\title{
Environments of ethidium binding to allosteric DNA: II. accessibility, mobility and mode of binding
}

\author{
Dongchul Suh ${ }^{1,2}$ \\ ${ }^{1}$ Department of Biochemistry, School of Medicine, \\ Pochon CHA University, Kyungki-Do 487-800, Korea \\ ${ }^{2}$ Corresponding author: Tel, +82-31-543-2654; \\ Fax, +82-31-543-2818; E-mail, DCSuh@hanmail.net
}

Accepted 14 November 2000

\begin{abstract}
DNA binding compounds were previously shown to bind to the right-handed DNA forms and hybrid B-Z forms in a highly cooperative manner and indicate that structural specificity plays a key role in a ligand binding to DNA. In this study, the modes of binding and structural specificity of agents to unusual DNA are examined by a variety of fluorescence techniques (intensity, polarization and quenching, etc.) to explore a reliable method to detect the association environment of ligands to deoxyoligonucleotides initially containing a B-Z junction between the lefthanded Z-DNA and right-handed B-DNA. The results of fluorescence energy transfer measurement demonstrated that the ligand molecules bind to the allosterically converted DNA structures by intercalation. In the absence of high-resolution structural data, this fluorescence energy transfer measurement allowed reliable measures and infer the binding environment of ligands to the allosteric DNA structures.
\end{abstract}

Keywords: allosteric DNA, fluorescence energy transfer, fluorescence polarization, fluorescence quenching, mode of binding

\section{Introduction}

The earlier study has defined the structural specificity of DNA binding ligands with allosteric DNA (Suh, 1999). The binding modes of ligands binding to a $B-Z$ junction and its allosterically converted DNA conformations were continuously investigated. Ethidium was again used for the simple DNA binding ligand. The highly cooperative ethidium binding to the allosterically converted DNA conformations was previously reported (Suh et al., 1991). The developments of novel drugs for DNA-mediated reactions are closely related to the sequence specificity of ligands with DNA (Chaires et al., 1996). The unusual
DNA structures were thought to provide the potential targets for drugs (Wells, 1988). The specific association of ligands with unusual DNA may provide useful information for such purpose (Suh et al., 1991). The unusual DNA structures of B-Z junction was reported as a local region of structurally distorted DNA (Sheardy, 1988). Understanding the nature of association between the interacting ligands to DNA is essential when the new agents for DNA binding are investigated for their target (Chaires et al., 1996). Furthermore, if the agents possesses sequence specific binding to the double-stranded DNA, the two most likely binding modes, intercalation or groove binding should be considered. The binding mode is most convincingly established by high-resolution structural studies, using either $x$-ray diffraction methods or NMR. Intercalation, in which a planar ligand moiety is inserted between adjacent base pairs, results in a substantial change in DNA structure. It causes the lengthening, stiffening, and unwinding of DNA helix (Waring, 1965). By intercalation, the planar chromophores are in close contact with the DNA base pairs, and are oriented roughly perpendicular to the DNA helix axis. Thus, fluorescence energy transfer methods may provide the way of determining the orientation of the chromophores of ligand and its proximity to the DNA bases (Suh and Chaires, 1995; Kelly and Barton, 1999).

The purpose of this study is to show how fluorescence methods can be used reliably to infer the environment of DNA-ligand complexes. The results in this study show that the standard fluorescence measurements, such as fluorescence intensity, polarization, and solute quenching studies may detect the environments of binding agents with DNA (Lakowicz, 1983). From these studies, fluorescence contact energy transfer and the intercalation from the groove binding clearly be established (LePecq and Paoletti, 1967). The results in this study will contribute to a convenient guide to establish the binding mode of new DNA binding agents.

\section{Materials and Methods}

\section{Materials}

A deoxyoligonucleotide, which is designated BZ-1, was described previously (Sheardy, 1988; Suh et al., 1991). The sequence of the double-stranded deoxyoligonucleotide, BZ-1 (C: 5-methyl-C) is as follows:

$$
\begin{aligned}
& \text { 5'-d[CGCGCGCGACTGACTG] } \\
& \text { 3'-d[GCGCGCGCTGACTGAC] }
\end{aligned}
$$


Each strand of linear DNA oligomers was mixed at 1:1 stoichiometric ratio. Then, the mixture was heated up to $90^{\circ} \mathrm{C}$ and slowly cooled down to $20^{\circ} \mathrm{C}$. It was annealed completely to form the duplex of non, self-complementary sequences. Ethidium and other reagents were purchased from Sigma (Sigma Chemical Co., St. Louis, $\mathrm{MO}$ ). Ethidium concentrations were determined spectroscopically by measuring the absorbance at $480 \mathrm{~nm}$ using $\varepsilon=5,600 \mathrm{M}^{-1} \mathrm{~cm}^{-1}$ (Waring, 1965). The experiments were performed in BPE buffer (so called, low salt) containing $6 \mathrm{mM} \mathrm{Na}_{2} \mathrm{HPO}_{4}, 2 \mathrm{mM} \mathrm{NaH}_{2} \mathrm{PO}_{4}$, and $1 \mathrm{mM}$ EDTA, pH 7.0. The BPE buffer plus $4.5 \mathrm{M} \mathrm{NaCl}$ (high salt) was prepared by adding $5 \mathrm{M}$ stock $\mathrm{NaCl}$ solution to the BPE buffer.

\section{Deoxyoligonucleotide BZ-1 was treated by exonu- clease III}

The upper strand of an oligonucleotide, BZ-1 was $5{ }^{\prime}-{ }^{32} \mathrm{P}$ end labeled with $\left[\gamma^{32} \mathrm{P}\right]$ ATP $(1000 \mathrm{Ci} / \mathrm{mmol}$, New England Nuclear) and T4 polynucleotide kinase according to standard procedures (Suh et al., 1997). Prior to exonuclease III treatment, each labeled DNA strand was annealed to its complementary strand (Suh et al., 1991). The labeled duplex oligomer, $3 \mu \mathrm{g}$ was treated with 0.1 , $1 \mathrm{U}$ of exonuclease III (Promega, $100 \mathrm{U} / \lambda$ ) at $37^{\circ} \mathrm{C}$ for $15 \mathrm{~min}$. Following the exonuclease III treatment, DNA samples were mixed with formaldehyde, loaded onto a $20 \%$ denaturing polyacrylamide sequencing gel. The gels $(40 \times 30 \times 0.8 \mathrm{~cm})$ were run for $2.5 \mathrm{~h}$ at $2,000 \mathrm{~V}$. Gels were covered with cellophane wrap, placed on $\mathrm{X}$ ray films overnight at $4^{\circ} \mathrm{C}$.

\section{Spectral measurement}

The corrected fluorescence spectra of ethidium were obtained using a photon counting fluorometer, Greg 200 (I.S.S., Champaign, IL). The corrected excitation spectra of ethidium were measured at $605 \mathrm{~nm}$ for the emission. All experiments were performed at room temperature. Absorbance spectra were measured using a Cary 219 spectrophotometer (Varian, Palo Alto, CA). The absorption spectra of compounds were measured in the presence and absence of DNA. Circular dichroism spectra were measured with a JASCO J-500A spectropolarimeter.

\section{Fluorescence polarization and fluorescence quen- ching}

The fluorescence polarization spectra of ethidium with DNA were measured at $20^{\circ} \mathrm{C}$ by four independent measurements of fluorescence intensity (Lakowicz, 1983). The steady state fluorescence polarization, $p$ was calculated

$$
p=\left(I_{v v} I_{h h}-I_{h v} I_{v h}\right) /\left(I_{v v} I_{h h}+I_{h v} I_{v h}\right)
$$

where the subscripts refer to the positions of polarizers for excitation and emission (v: vertical, h: horizontal). The fluorescence polarization spectra of ethidium with allosteric DNA forms were plotted as a function of wavelength.

Fluorescence quenching experiments were performed in order to reveal the accessibility of bound ligands in DNA. The ratio of fluorescence intensity was presented to construct the Stern-Volmer plots according to the equation (Eftink, 1991)

$$
\mathrm{F}_{\mathrm{o}} / \mathrm{F}=1+\mathrm{K}_{\mathrm{q}} \mathrm{t}_{\mathrm{o}}[\mathrm{Q}]
$$

where $F_{0}$ and $F$ represent the fluorescence intensity in the absence and presence of DNA, respectively, the product of $\mathrm{K}_{\mathrm{q}}$ is the Stern-Volmer quenching constant in which $\mathrm{K}_{\mathrm{q}}$ is the quenching constant and $\mathrm{t}_{\mathrm{o}}$ is the fluorescence decay time in the absence of quenchers. [Q] represents the concentration of quenchers.

\section{Measurement of fluorescence contact energy trans- fer}

Fluorescence contact energy transfer is known to occur by transferring the energy of the excited state from a donor to an acceptor (Clegg, 1992). The ratio of quantum yield can be corrected by the quantum yield at 310 $\mathrm{nm}$ which DNA has little absorbance at the same ligand concentration,

$$
Q_{\lambda} / Q_{310}=\left(q_{b} / q_{f}\right) /\left(q_{b} / q_{f}\right)_{310}
$$

where in $q=F / \varepsilon, q$ is the quantum yield, $F$ is the fluorescence intensity and $\varepsilon$ is the molar extinction coefficient. $B$ and $f$ refer to the bound and free state, respectively. It should be noted that the absorbance of the ligandDNA complexes were measured by maintaining the same concentration of DNA for the reference solutions in order to exclude the contribution of the absorbance of DNA over the UV range (Suh and Chaires, 1995). The spectra were converted into the profiles of extinction coefficients and the ratio of extinction coefficients in the absence and presence of DNA were estimated. The fluorescence intensity of ethidium bromide in the absence and presence of DNA were measured independently using a photon counting fluorometer which can give the corrected excitation spectra. The fluorescence intensity of ethidium bromide with DNA was particularly calibrated by the inner filter effect (Suh, 1999). The ratio of quantum yield can be calculated and plot over the range of UV wavelength.

\section{Results and Discussion}

\section{Allosteric conversion of DNA forms measured by CD}

The conformations of allosteric DNA forms were examined by circular dichroism spectroscopy. In the 


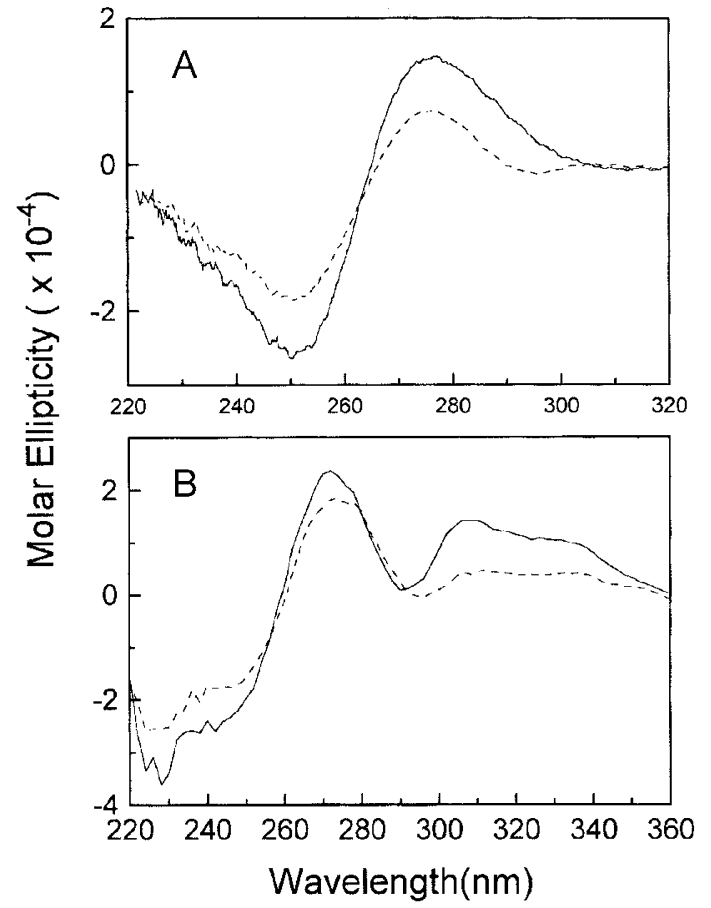

Figure 1. (A) $C D$ spectrum represents the right-handed $B$-form and the $B-$ $Z$ form of BZ-1. (B) The allosterically converted DNA forms with ethidium molecules. The solid lines represent CD spectra in low salt and the dotted lines represent $C D$ spectra in high salt.

absence of ethidium, BZ-1 is in the B-form DNA in low salt and in the BZ hybrid DNA form in high salt (Figure $1 A)$. In the presence of ethidium, however, the spectra represent the DNA structures of ethidium-saturated oligomer in low- and in high salt by the allosteric conversion, in Figure 1B. Particularly for the DNAethidium complexes in low salt, there is a distinct $C D$ band, which is presumably related to the clustering of bound ligands (Chirico et al., 1990; Suh et al., 1991). The environments of bound ligands in allosteric DNA structures were examined by several fluorescence techniques.

\section{The band position within the sequence of $\mathrm{BZ}-1$ treated by exonuclease III}

The labeled strand was annealed to the complementary oligonucleotide to generate DNA construct. Each strand of the labeled oligonucleotide BZ-1 showed a different mobility in danaturing polyacrylamide gel depending on the sequence difference (data not shown). The cleavage pattern of the exonuclease III treatment for BZ-1 is shown in Figure 2. The autoradiogram indicates the sequence of the labeled upper strand of BZ-1 while the intensity of each position is varied by different response to exonuclease III.

Accessibility and fluorescence characteristics of ethidium with allosteric DNA

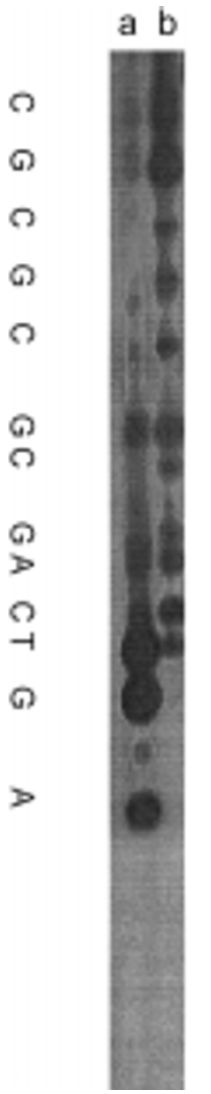

Figure 2. Autoradiogram of a deoxyoligonucleotide BZ-1 treated by the exonuclease III. The sequence is presented on the left. The products resulting from exonuclease III cleavage of BZ-1 by different amount of exonuclease III are represented at lane $\mathrm{a}(1 \mathrm{U})$ and $\mathrm{b}(0.1 \mathrm{U})$.

Fluorescence quenching can be used for monitoring the accessibility of bound fluorophores to the quenching agents. Fluorescence quenching refers to any process that decreases the fluorescence intensity of a given compound by the effect of quenchers (Barkley et al., 1991). Quenching was measured for the bound ethidium in BZ-1 under the low- and high-salt condition, which are shown in Figure 3A. As a reference, free molecules of ethidium can easily be exposed to the quenching agents. The Stern-Volmer plots were obtained for the iodide quenching of bound ethidium with allosteric DNA forms of BZ-1. The ratio, $\mathrm{I}_{\mathrm{o}} / \mathrm{l}$, represents the value of fluorescence intensity of fluorophore in the absence and presence of a quenching agent (Eftink, 1991). The SternVolmer constant of free ethidium is $2.12 \mathrm{M}^{-1}$. The constant of bound ethidium to allosteric DNA forms of BZ1 was found to be 0.0 . The fluorescence of the intercalated ligands, daunomycin in other quenching studies, does not change in the presence of anionic quenching agent, such as $\mathrm{I}^{-}$(Chaires, 1983). Thus, the bound ligands with allosteric DNA forms of BZ-1 were completely inaccessible to the added quenching agents. This result demonstrates that ethidium is sequestered away from the solvent in allosteric DNA complexes and suggests 

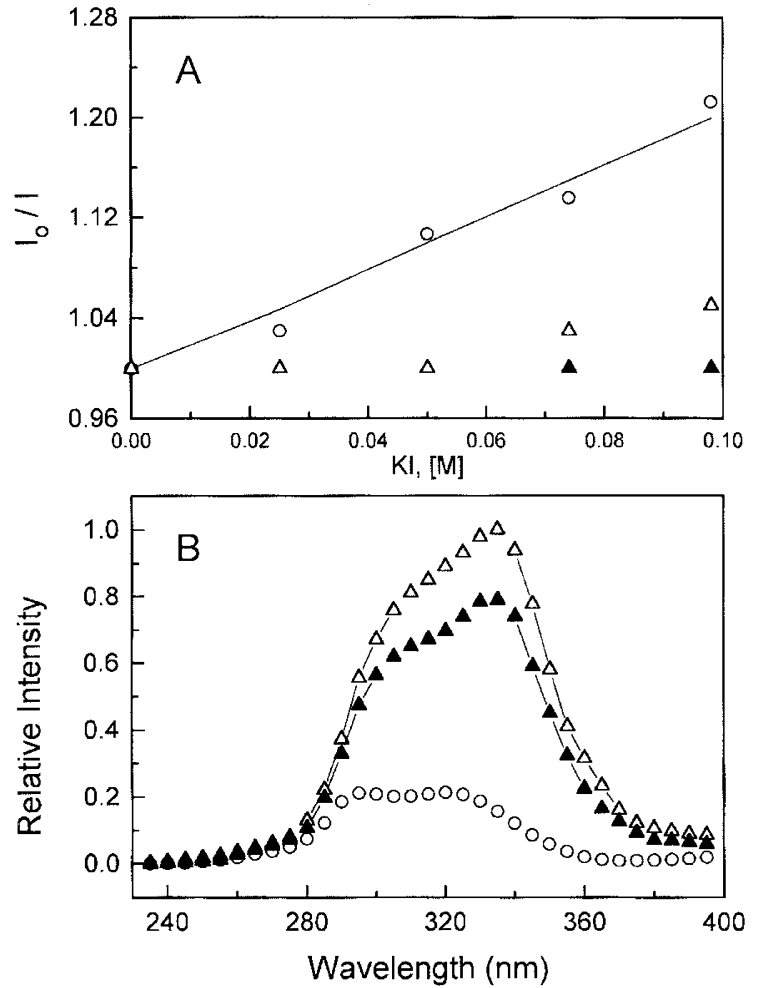

Figure 3. (A) The Stern-Volmer plots of iodide quenching for ethidium molecules (binding ratio $=0.02$ ). The line was fitted to the least-squares equation (correlation=0.99). (B) The corrected excitation fluorescence spectra of ethidium molecules. The symbols represent that the free ethidium molecules (0), the bound ethidium with the allosteric DNA forms in low (empty triangles), in high salt (filled triangles).

intercalative binding of ethidium.

The fluorescence excitation spectra were shown in Figure 3B. These spectra represent the fluorescence properties of ethidium in the absence and presence of allosteric DNA. The maximum position of the excitation spectrum shifts by about $20 \mathrm{~nm}$ upon ethidium binding to BZ-1. The magnitude of fluorescence was enhanced by ethidium binding to BZ-1 in the salt. Upon ethidium binding to DNA, the magnitude of fluorescence was 34 times enhanced. This result represents that the increase of quantum yield in the region of excitation suggests the energy transfer between DNA bases and bound fluorophores.

\section{The internal motion of bound ligands with allosteric DNA structures}

The fluorescence polarization spectra of ethidium for the excitation were shown in Figure 4. By steady-state fluorescence polarization, the rotational diffusion of ligands related to the binding reaction can be measured. The long-axis polarized rotational transition of ethidium molecule, which is parallel to the long axis of the phenyl group, occurs in the region of visible light (Hard and

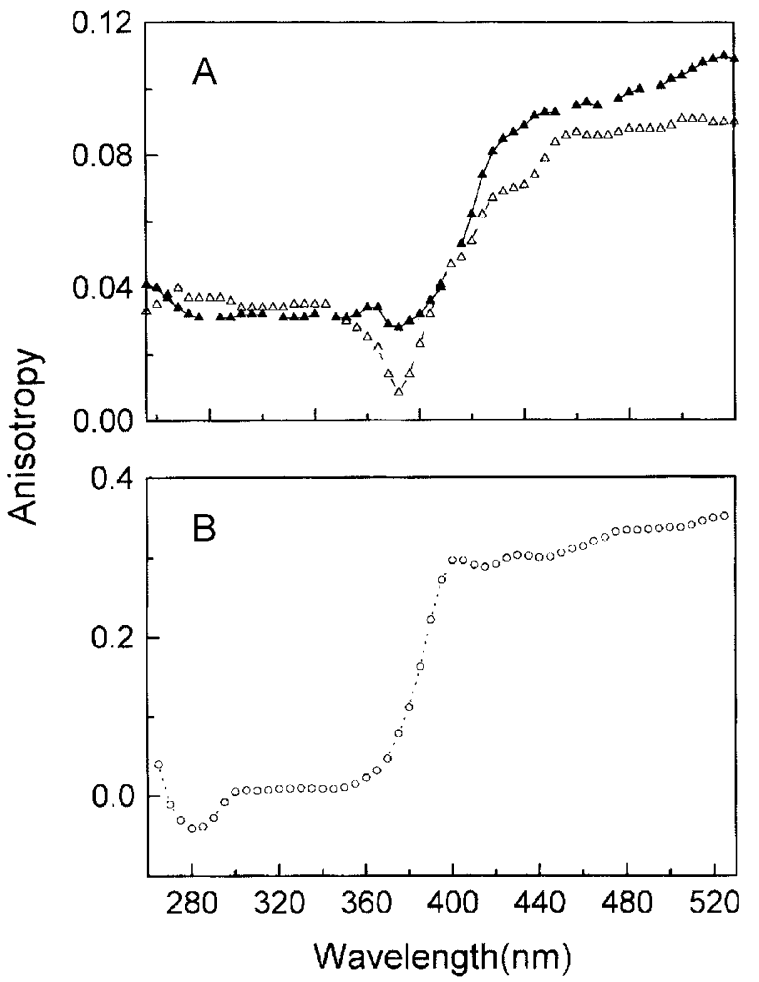

Figure 4. Excitation fluorescence polarization spectra of ethidium in the presence of allosteric DNA forms (A). Fluorescence polarization spectrum of free ethidium molecules was measured in $90 \%$ glycerol (B). The symbols represent that the free ethidium molecules(0), the bound ethidium with the allosteric DNA forms of BZ-1 in low (empty triangles) and in high salt (filled triangles).

Kearns, 1986). In this study, the long-axis polarized transition of free ethidium is shown in Figure 4B. In the range of $280-300 \mathrm{~nm}$, the polarization indicates negative when free ethidium is in $90 \%$ glycerol. The long-axis polarized transition at $270-320 \mathrm{~nm}$ of free ethidium changes to increase its magnitude when ethidium bound to BZ-1. Particularly, the short-axis polarized transition of free ethidium was demonstrated at the long wavelength (500-540 nm). It changes dramatically to decrease upon ethidium binding to BZ-1. Thus, the short-axis polarized band is very much depolarized. The internal motion and ligand wobbling contribute to the depolarization (Hard and Kearns, 1986). These results imply that the bound ligands in allosteric DNA in salt are immobilized since their polarization is increased as shown in Figure 4A.

\section{Shapes of ethidium-allosteric DNA complexes}

Perrin's law of depolarization predicts a linear relationship between fluorescence polarization and viscosity changes on temperature (Lakowicz, 1983). From the analyzed results of Perrin plot, certain information of shapes, such as the molar volume of fluorophore in DNA complex can be obtained. The Perrin equation for the excitation with polarized light is as follows: 

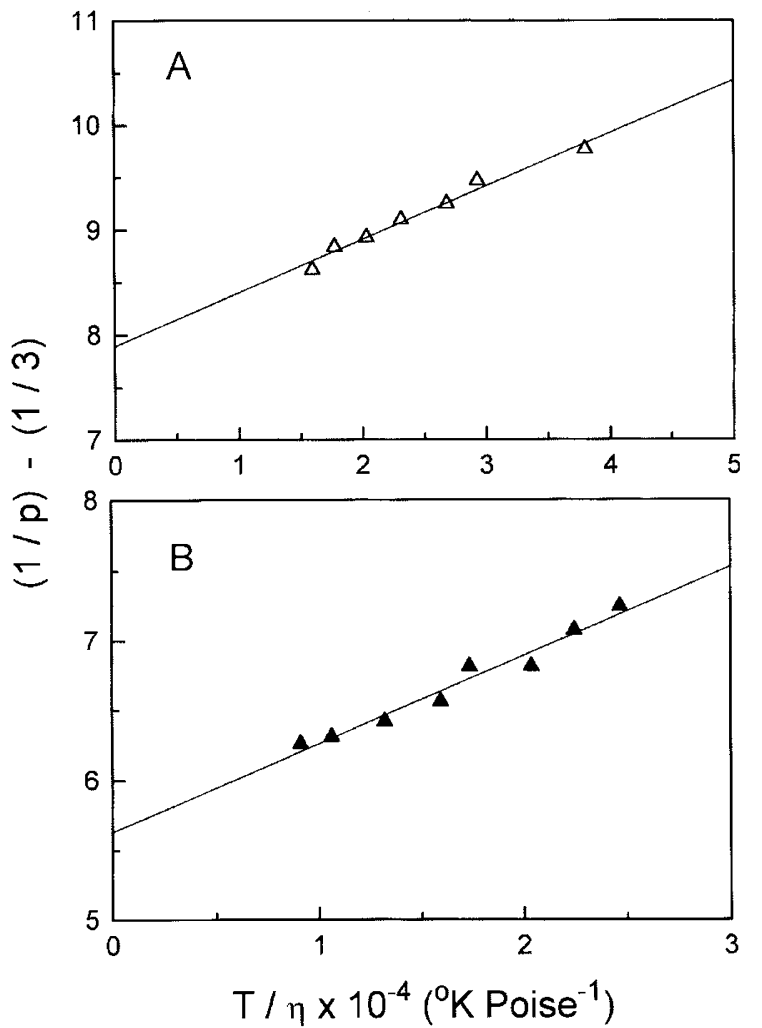

Figure 5. Perrin plots of ethidium binding to $B Z-1$ in low $(A)$ and high (B) salt, respectively. The changes of viscosity were monitored in the range of temperature $1-35^{\circ} \mathrm{C}$. The slope of Perrin plot for ethidium-BZ1 complex is $0.51 \times 10^{-4}(\mathrm{~A})$ and $0.64 \times 10^{-4}(\mathrm{~B})$, and the $\mathrm{y}$-intercept is $7.9(\mathrm{~A})$, and 5.6 (B). The value of $P_{0}$ is $0.12(A)$, and $0.17(B)$, respectively.

$$
(1 / P-1 / 3)=\left(1 / P_{\circ}-1 / 3\right)+\left(1 / P_{\circ}-1 / 3\right)(R \tau / V)(T / \eta)
$$

where $\mathrm{P}_{\mathrm{o}}$ is the limiting polarization in the absence of bound ligands; $R$ is the gas constant; $T$ is the absolute temperature; $\eta$ is the solvent viscosity(poise); $V$ is the molar volume of the rotating unit $\left(\mathrm{cm}^{3} \mathrm{~mol}^{-1}\right) ; \tau$ is the life time $\left(\mathrm{s}^{-1}\right)$. Perrin plots were shown in Figure 5. The slopes and intercepts representing the parameters of Perrin's law were obtained from these plots (summarized in Figure legends). The binding ratio was selected at the ratio of 20-bp per a ligand. The viscosity of water was assumed for the solution of low- and high-salt condition.

If the slope of Perrin plots can be put $\beta=(R \tau / V)$, the harmonic mean $(\lambda)$ of the rotational relaxation time is defined as, $\lambda=(3 \eta V) /(R T)$ (Lakowicz, 1983). The molar volume was obtained from both this relation and the parameters of Perrins law: $264,025 \mathrm{~cm}^{3} \mathrm{~mol}^{-1}$ in low salt, $149,745 \mathrm{~cm}^{3} \mathrm{~mol}^{-1}$ in high salt, respectively. This result suggests that the ethidium-DNA complexes in high salt are more compact than the complexes in low salt. Thus, the shape(s) of allosteric DNA with bound ligand is different.

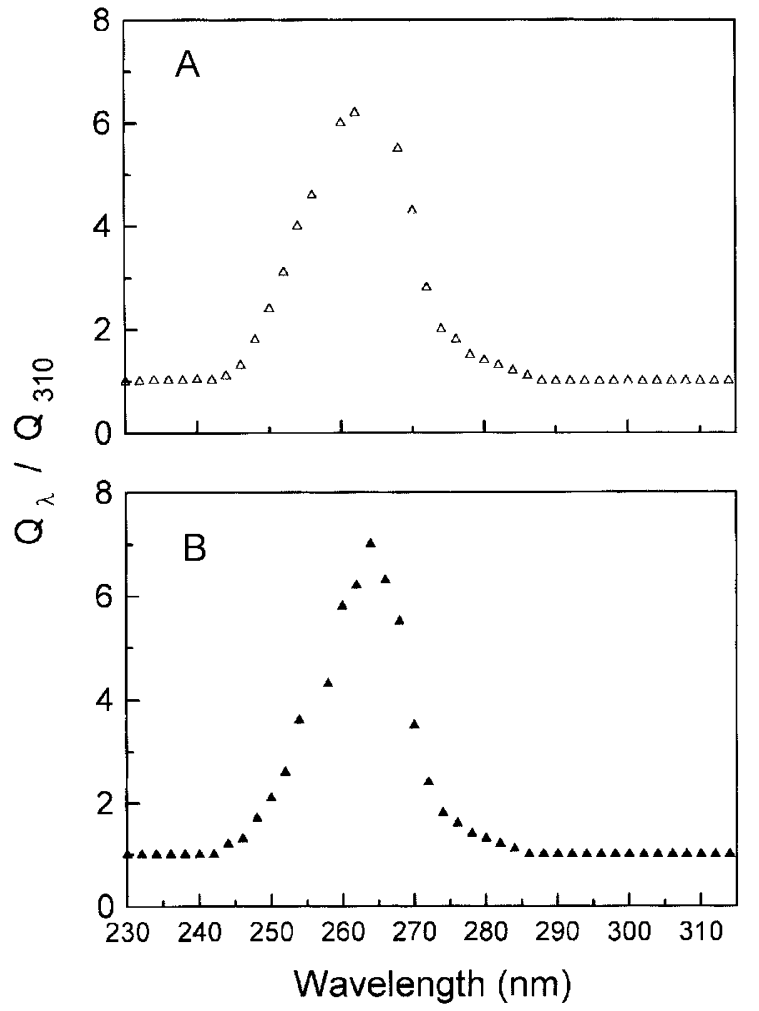

Figure 6. The ratio of the fluorescence quantum yield is shown for the bound ethidium molecules in allosteric DNA complexes as a function of the wavelength, in low $(A)$ and high salt $(B)$ (binding ratio $=0.1$ ).

\section{The mode of binding was determined by fluorescence contact energy transfer}

Fluorescence energy transfer is occurred generally from DNA bases to the bound ligands. The magnitudes of contact energy transfer directly from the base pairs of DNA to the bound ligands can be measured by the ratio of the relative quantum yield of ligands-DNA complexes (LePecq and Paoletti, 1967). The quantum yield of bound ligands increases at the excitation wavelength in concomitant with the absorbance of DNA. The ratio $\mathrm{q}_{\mathrm{b}} /$ $q_{f}$, quantum efficiency of bound ethidium in DNA divided by that of free ethidium, can be obtained. The corrected excitation spectrum and the UV absorbance spectrum of DNA were obtained in the region of wavelengths corresponding to DNA absorbance. The wavelength $310 \mathrm{~nm}$ was chosen for the reference because DNA has little absorbance at this wavelength. The plots of contact energy transfer are shown as a function of wavelengths in Figure 6. The results in this study demonstrate that DNA bases and bound ligands are in close contacts (Scaria and Shafer, 1991). The quantum yield of bound ethidium molecules in the allosteric DNA forms of BZ-1 increases dramatically at $260 \mathrm{~nm}$. The efficiency of quantum yield for the allosteric DNA with bound ethidium is above 6 . The results indicate that the ethidium 
molecules bind to allosteric DNA by intercalation.

\section{Acknowledgement}

This work was supported by the Genetic Engineering research grant (1998-019-D00083) from the Korean Ministry of Education. BZ-1 was kindly given by Dr. Sheardy in Seton Hall University (USA). I would like to thank Dr. Chaires in the University of Mississippi Medical Center (USA) for helpful discussion.

\section{References}

Barkley, M. D., Thomas, T. J., Maskos, K. and Remers, W. A. (1991) Steady-state fluorescence and molecular-modeling studies of tomaymycin-DNA adducts. Biochemistry 30: 44214431

Chaires, J. B. (1983) Equilibrium studies on the interaction of daunomycin with deoxyoligonucleotides. Biochemistry 22: 4204-4211

Chaires, J. B., Satyanalayana, S., Suh, D., Fokt, I., Przewloka, T. and Priebe, W. (1996) Parsing the free energy of anthracycline antibiotic binding to DNA. Biochemistry 35: 20472053

Chirico, G., Lunelli, L. and Baldini, G. (1990) Conformation of Intercalated DNA Plasmids Investigated by Circular Dichroism and Dynamic Light Scattering. Biophys. Chem. 38: 201-211

Clegg, R. M. (1992) Fluorescence resonance energy transfer and nucleic acids. Meth. In Enzymol. 211: 353-389. Academic Press, N.Y.

Eftink, M. R. (1991) in Topics in Fluorescence Spectroscopy (Lakowicz, Ed.) Vol. 2, pp. 53-127, Plenum Press, New York.

Hard, T. and Kearns, D. R. (1986) Anisotropic motions in intercalative DNA-dye complexes. J. Phys. Chem. 90: 34373444

Hard, T. and Kearns, D. R. (1986) Anisotropic overall and internal motions of short DNA fragments. Nucl. A. Res. 14: 3945-3956

Kelly, S. O. and Barton, J. K. (1999) Electron transfer between bases in double helical DNA. Science 283: 375-381

Lakowicz, J. R. (1983) Principles of Fluorescence Spectroscopy, Plenum Press, N.Y.

LePecq, J.-B. and Paoletti, C. (1967) A fluorescent complex between ethidium bromide and nucleic acids. J. Mol. Biol. 27: 87-106

Scaria, P. V. and Shafer R. H. (1991) Binding of ethidium bromide to a DNA triple helix. J. Biol. Chem. 266: 5417-5423

Sheardy, R. D. (1988). Preliminary Spectroscopic Characterizations of a Synthetic DNA Oligomer Containing a B-Z Junction at High Salt. Nucleic Acids Res. 16: 1153-1167

Suh, D., Sheardy, R. D. and Chaires, J. B. (1991) Unusual Binding of Ethidium to a DNA Containing a B-Z Junction. Biochemistry 30: 8722-8726

Suh, D. and Chaires, J. B. (1995) Criteria for the Mode of Binding of DNA Binding Agents. Bioorg. Med. Chem. 3: 723728

Suh D., Wilson III, D. M. and Povirk L. F. (1997) 3'-Phosphodiesterase activity of human apurinic/apyrimidinic endonuclease at DNA double-strand break ends. Nucleic Acids Res. 25: 2495-2500

Suh, D. (1999) Cooperative binding interaction of ethidium with allosteric DNA. Exp. Mol. Med. 31: 151-158.

Waring, M. J. (1965) Complex formation between ethidium bromide and nucleic acids. J. Mol. Biol. 13: 269-282

Wells, R. D. (1988) Unusual DNA Structures. J. Biol. Chem. 263: $1095-1098$ 